International Journal of Advanced Astronomy, 2(2)(2014) 34-42
International Journal of Advanced Astronomy
SPC
Journal home page: $\begin{gathered}\text { www. sciencepubco.com/index.php/IJAA } \\ \text { doi.14419/ijaa.v2i2.3585 } \\ \text { Review Paper }\end{gathered}$

\title{
On the evolving and emitting quantum black holes and the scale independent black hole cosmology
}

\author{
U. V. S. Seshavatharam ${ }^{1 *}$, S. Lakshminarayana ${ }^{2}$ \\ ${ }^{1}$ Honorary faculty, I-SERVE, Alakapuri, Hyderabad-35,AP, India \\ ${ }^{2}$ Dept. of Nuclear Physics, Andhra University, Visakhapatnam-03,AP, India \\ *Corresponding author E-mail: seshavatharam.uvs@gmail.com
}

\begin{abstract}
Along with presently the believed black holes that are expected to be formed by gravitational collapse of a massive star, there may exist primordial evolving black holes. By absorbing the hidden vacuum energy primordial evolving black holes become massive. Planck mass can be considered as the basic mass of any black hole and can be called as the baby Planckion. Very high temperature of the baby Planckion is the only heat source for the evolving black holes or evolving Planckion's decreasing temperature. Considering the current cosmic microwave back ground temperature as a quantum gravitational effect of the evolving primordial cosmic black hole, general theory of relativity and quantum mechanics can be combined into a 'scale independent' true unified model of quantum gravity. Considering cosmic black hole's light speed rotation and galactic revolution, observed galactic rotational curves can be understood. In the past, decreasing high cosmic black hole temperature forced hydrogen atom to emit increasing photon energy resulting in the observed redshift. Aged super novae dimming may be due to the effect of past high cosmic black hole temperature. As cosmic time passes, decreasing current cosmic black hole temperature makes hydrogen atom to emit increased quanta of energy causing the future redshift. In future, with reference to the laboratory hydrogen atom, decreasing current cosmic temperature and measured rate of increase in emitted photon energy - true rate of future cosmic expansion can be understood. With reference to the decreasing current Hubble constant and decreasing current cosmic black hole temperature, true rate of future cosmic expansion can also be understood. Foundations of Quantum mechanics and General theory of relativity may be reviewed in this unified way.
\end{abstract}

Keywords: Quantum Gravity, Evolving Black Holes, Geometric Horizon, Standard Cosmology, Black Hole Cosmology, CMBR Isotropy and Anisotropy, Redshift, Galactic Rotational Curves.

\section{Introduction}

History of modern cosmology is very interesting. At first in 1916 Einstein proposed an intellectual but unsuccessful static model of cosmology with the famous 'lambda term' and science community forced him to abandon the term. Later in 1920s Friedmann proposed an expanding model of cosmology and was recognized only after Hubble's work on the galactic redshift (Hubble, E.P 1947) Without reaching any other part of the universe, Friedmann boldly proposed that universe looks the same from any part of the universe! In this regard in 1988 S.W. Hawking suggested that, there is no scientific evidence to Friedmann's second assumption and it is being believed only on modesty (Hawking S.W 1988, p.25). Very unfortunate thing is that, so far science and technology could not provide a single clue in support of this assumption. If so, one can certainly doubt the output physics and consequences of Friedmann cosmology. In 1948 Fred Hoyle proposed 'steady state cosmology' and was found to be insightful. At the same time in 1948 Gamow proposed hot big bang model of expanding cosmology and was not recognized by the science community (Alpher R.A et al 1948). In 1964 unexpectedly hot big bang model got a great evidence (A. Penzias 1978). In 2000, cosmologists again unexpectedly proposed accelerating model of cosmology with distant super novae dimming against a normally expected 'decelerating model of hot big bang'. Most surprising thing is that so far no telescope or particle accelerator provided evidence to the indirectly confirmed 'dark energy' of the accelerating model of the universe. Another interesting thing is that, the abandoned lambda term has been reconsidered by the science community to understand the existence of dark energy. In this long journey the very interesting thing is that, the subject of cosmology was largely influenced by Hubble's interpretations on galactic redshift. Here the authors would like to stress the fact that, without measuring and confirming the 'actual' galaxy receding, it may not be reasonable to confirm the Hubble's redshift interpretation, the current cosmic acceleration and the existence of dark energy. In 1947 Hubble himself thought for an alternative explanation for understanding the observed galactic redshifts. Another unfortunate thing is that so far no single parameter of modern cosmology has been obtained from the standard microscopic physics. Since 1980 s 'cosmic inflation' is so widely accepted that it is often taken as an established fact. The idea is that the geometry and uniformity of the cosmos were established during an intense early growth spurt. But very shocking news is that, some of the creators of the inflation theory, like Paul J. Steinhardt, are having second thoughts. As the original theory has been developed, cracks have appeared in its logical foundations (Steinhardt, Paul J 2011). Various proposals are being circulated for ways to fix or replace it. Quantum cosmology is a field attempting to study the effect of quantum mechanics on the formation of the universe, or its early evolution, especially just after the Big Bang. It can be suggested that the subject of modern cosmology (Saul 
Perlmutter 2011, David N. Spergel et al 2013) is completely based on distant observations and is open for in-depth discussion.

\section{Three great facts of cosmology and to reinterpret the galactic redshift}

The 3 great facts of cosmology were: galactic redshift, cosmic microwave background radiation temperature and super novae dimming. It is very important to note that, 1) If it is true that galaxy constitutes so many stars, each star constitutes so many hydrogen atoms and light is coming from the excited electron of galactic hydrogen atom, then considering redshift as an index of 'whole galaxy' receding may not be reasonable. 2) Increased redshifts and increased distances forced Edwin Hubble to propose the Hubble's law. Even then, merely by estimating galaxy distance and without measuring galaxy receding speed, one cannot verify its acceleration. Clearly speaking: two mistakes are being possible here. i) Assumed galaxy receding speed is not being measured and not being confirmed. ii) Without measuring and confirming the galaxy receding speed, how can one say and confirm that its receding speed is increasing and accelerating? 3) If light is coming from the atoms of the gigantic galaxy, then instead of wavelength difference, in terms of 'quantum of energy' redshift can also be interpreted as an index of the galactic cosmological atomic 'light emission mechanism'. 4) According to standard cosmology, since decoupling, the temperature of the CMBR temperature has dropped by a factor of roughly 1100 due to the expansion of the universe. As the universe expands, the CMB photons are redshifted, making the radiation's temperature inversely proportional to a parameter called the universe's scale factor. If $T_{t}$ is the temperature of the $\mathrm{CMB}$ and $z$ is the observed redshift, then $T_{t} \cong(1+z) 2.725 \mathrm{~K}$ where $(1+z)$ is known as the universal scale factor. Extending this concept, it can also be possible to guess that emitted quantum of energy is inversely proportional to the cosmic back ground temperature. If so aged and distant super novae dimming effect can also be considered as a result of high CBR temperature. In 1947 Hubble himself stated that (Hubble, E.P 1947 p.166-167): "We may predict with confidence that the 200 inch will tell us whether the red shifts must be accepted as evidence of a rapidly expanding universe, or attributed to some new principle in nature. Whatever may be the answer, the result may be welcomed as another major contribution to the exploration of the universe".

\subsection{Black hole cosmology - the scale independent true quantum gravity replica}

Most of the modern physicists believe that, 1) Quantum gravitational effects (Ashtekar 1986, Hawking S W 1987, Carlo Rovelli 2011, Schwarz, John H 2007, Maité Dupuis et al 2012) are extremely weak and cannot be tested in any ground based laboratory operating under low energy scales. 2) As the laboratory experimental energy scale increases and approaches the Planck scale, quantum gravitational effects become stronger and their results can be observed and measured. Some of the other modern physicists believe that, during the cosmic evolution, Planck scale quantum gravitational interactions might have an observable effect on the current observable cosmological phenomena. In this context, the later proposal seems to have more impact on the observable universal laboratory. Clearly speaking, with respect to the Planck scale early universal laboratory, current universe can be considered as a low energy scale laboratory. If so, cosmological quantum gravity can be considered as the scale independent model. If one is willing to consider the current observable universe as a low energy scale operating laboratory, currently believed cosmic microwave back ground temperature can be considered as the low energy quantum gravitational effect. At any time in the past, i.e as the operating energy scale was assumed to be increasing; past high cosmic back ground temperature can be considered as the high energy quantum gravitational effect. Thinking in this way, starting from the Planck scale and with reference to the decreasing magnitude of cosmic back ground temperature, quantum gravity can be considered as a scale independent model and the universe can be considered as the best quantum gravitational object.

\section{Major shortcomings of modern cosmology}

The basic shortcomings of modern cosmology can be expressed as follows.

1) No direct observational evidence to Friedmann's second assumption. We believe it only on the grounds of modesty. Really if there was a 'big bang' in the past, with reference to formation of the big bang as predicted by general theory of relativity and with reference to the cosmic expansion that takes place simultaneously in all directions at a uniform rate at that time about the point of big bang - 'point' of big bang can be considered as the centre or characteristic reference point of cosmic expansion in all directions. In this case, saying that there is no preferred direction in the expanding universe - may not be correct.

2) When Friedmann's cosmology was taking its final shape, black hole physics was in its beginning stage. Recent observations confirm the light speed rotation of black holes. So far no theoretical proof is available for cosmic non-rotation. So far no experimental or observational evidence is available for super luminal rotation speed of any celestial object. By considering 'black hole geometry' as the 'eternal cosmic geometry' and by assuming 'constant light speed rotation' with Hubble constant as angular velocity, throughout the cosmic evolution, at any time the currently believed cosmic 'critical density' can be shown to be the cosmic black hole's eternal 'mass density'. If so it is possible to suggest that, there is no theoretical base in Friedmann's 'critical density' concept and the 'matter density' classification scheme. Clearly speaking, when the currently believed 'critical density' itself represents the mass density of a light speed rotating black hole universe and as there is no observational or experimental evidence to Friedmann's second assumption, the density classification scheme of Friedmann cosmology must be reviewed at fundamental level.

3) No theoretical base in considering the Hubble's constant merely as the cosmic expansion parameter. With coefficient of unity, if one is willing to consider $\left(c / H_{0}\right)$ as a characteristic length, then based on elementary dimensional analysis it is very simple to show that, dimensions of $H_{t}$ are $\mathrm{rad} / \mathrm{sec}$ and thus with a coefficient of unity and with reference to the characteristic light speed, $H_{t}$ can be considered as cosmic angular velocity. Note that, in any case if length coefficient is less than unity or greater than unity, 'Hubble length' may lose its physical identity.

4) 'Rate of decrease in current 'Hubble's constant' can be considered as a measure of current cosmic 'rate of expansion'. If rate of decrease in current 'Hubble's constant is very small and is beyond the scope of current experimental verification, then the two possible states are: a) current 'Hubble's constant is decreasing at a very slow rate and current universe is expanding at a very slow rate and $b$ ) at present there is no 'observable' cosmic expansion or acceleration. The same approach can be applied to the current CMBR temperature.

5) By substituting the geometric mean mass of $\left(c^{3} / 2 \mathrm{GH}_{0}\right)$ and $\sqrt{\hbar c / G}$ in the famous Hawking's black hole temperature formula automatically the observed $2.725 \mathrm{~K}$ can be fitted very accurately (David N. Spergel et al 2013, Hawking SW 1975). Standard cosmology is silent in this regard.

6) No comparative and relational study in between Friedmann cosmology, Mach's principle and microscopic physical phenomena. 
7) No direct observational evidence to the current galaxy receding and current cosmic expansion. Similarly no direct observational evidence for the current cosmic acceleration and the dark energy.

\section{Evolving black holes and their geometric horizons}

Very recently S.W. Hawking modified his Black hole theory (Hawking SW 2014) with "Apparent horizons". This brought a serious confusion among the black hole physicists and whole science community. In his words: "There is no escape from a black hole in classical theory. Quantum theory, however, enables energy and information to escape from a black hole". He admits that, a full explanation of the process would require a theory that successfully merges gravity with the other fundamental forces of nature. But that is a goal that has eluded physicists for nearly a century. However 'the correct treatment'- 'remains a mystery'. Abhas Mitra (Mitra A 2000) has shown that true black holes can never form. In his opinion the so-called black holes observed by astronomers are actually radiation pressure supported Eternally Collapsing Objects (ECOs). These balls of fire are so hot that even neutrons and protons melt there and whose outward radiation pressure balances the inward pull of gravity to arrest a catastrophic collapse before any Black Hole or 'singularity' would actually form.

Now the fundamental question to be answered is- Will any black hole exists without its event horizon? In author's opinion - the answer is "yes". By considering Hawking's view and Abhas Mitra's view, if one is willing to replace the "event horizon" with "geometric horizon", black holes can be considered as real "very hot celestial quantum objects' with emission of electromagnetic energy under extreme gravitational attraction. Along with the presently believed black holes that are expected to be formed by gravitational collapse of a massive star, there may exist primordial very hot evolving black holes and their origin may be interlinked with the Planck scale. During their evolution, by absorbing the hidden vacuum energy primordial evolving black holes become massive. As they grow their temperature will decrease. Clearly speaking their temperature is inversely proportional to the square root of their mass and the Planck mass. Whereas, with reference to hawking black hole's temperature formula, collapsing black hole's temperature is inversely proportional to the mass of the black hole only. This is a very important point to be noted. Baby Planckion's high temperature was the only heat source for the evolving Planckions' decreasing temperature. Extending this proposal, the current universe can be considered as a huge evolving black hole of radius equal to the current Hubble length and temperature equal to the current cosmic microwave back ground temperature 2.725 kelvin. In reality one may see or may not see a black hole, but by considering the whole 'observable universe' as a huge primordial evolving black hole, many interesting things will come into review.

\subsection{To understand the growing geometric boundary of a growing black hole}

Generally any living or non-living object is being identified by its shape. In our daily life generally it is observed that any animal or fruit or human being (from birth to death) grows with closed boundaries (irregular shapes also can have a closed boundary). An apple grows like an apple. An elephant grows like an elephant. A plant grows like a plant. A human being grows like a human being. As their shape is being maintained continuously throughout their life time they won't change their respective identities. These are the observed biological facts. From these observed facts it can be suggested that "growth" or "expansion" can be possible with a closed boundary. Thinking that nature loves symmetry, in a heuristic approach in this paper authors assume that any black hole possesses a (growing) structural boundary by which its physical structure always seems to be identified as a (growing) black hole. Such type of boundary can be called as the growing geometric boundary of the growing black hole. Planck mass can be considered as the primordial very hot baby black hole. It can be considered as the seed of any growing black hole and can be called as the baby Planckion.

\subsection{Natural non-escaping of a freely falling body}

A freely falling body attains light speed when it reaches the black hole surface. At the same time it completely loses its shape and size due to black holes surface gravity and high temperature. The moment it reaches the light speed, (in a highly deformed state) it stats escaping from the black hole geometric horizon. Due to high surface gravity, its light speed escape velocity becomes zero within a short span. By any strange control mechanism if it is able to maintain its shape, size and light speed (against the black hole surface gravity and temperature), then certainly it will escape from the black hole geometric horizon.

\subsection{Natural escaping of photon}

Being a quantum mechanical object even though surface gravity is high photon will escape from the massive Planckion's geometric horizon. Clearly speaking during its escape from the massive Planckion's geometric horizon, photon may lose energy due to massive Planckion's surface gravity and show gravitational redshift but it will not lose its speed. Thus with increasing redshift photon will continue its journey until its energy becomes zero and redshift reaches infinity. For a photon moving towards the massive Planckion's horizon, its speed remaining constant it experiences gravitational blue shift and again speed remaining constant it leaves the massive Planckion's horizon by losing its acquired energy by gravitational redshift. Compared to the photon that originates from the black hole, photon that enters and leaves the black hole will make a long journey.

\section{The classical limits of force and power}

Without considering the current notion of black hole physics, Schwarzschild radius of black hole can be estimated with the characteristic limiting force of magnitude $\left(c^{4} / G\right)$. The outstanding problem in particle physics today is the inclusion of gravity in a single, unified quantum theory of all the fundamental interactions. Particle physicists have long suggested that the four observed fundamental forces of nature (the gravitational, electromagnetic, weak nuclear and strong nuclear forces) are separate, low energy manifestations of what was once a single force at times close to the Big Bang. It is postulated that as the universe expanded and cooled, this single force gradually broke down into the four separate interactions as observed today. However, unification theories that seek to unify the force of gravity with all the other forces (Theories of Everything) remain elusive, as the gravitational interaction lacks a quantum formulation.

To unify cosmology, quantum mechanics and the four observed fundamental cosmological interactions - certainly a 'unified force' is required. In this connection $\left(c^{4} / G\right)$ can be considered as the classical force limit. Similarly $\left(c^{5} / G\right)$ can be considered as the classical power limit. If it is true that $c$ and $G$ are fundamental physical constants in physics, then $\left(c^{4} / G\right)$ and $\left(c^{5} / G\right)$ can also be considered as fundamental compound physical constants. These classical limits are more powerful than the Uncertainty limit. Note that by considering the classical force limit $\left(c^{4} / G\right)$, the famous

Planck mass can be obtained. 
5.1. Simple applications of $\left(c^{4} / G\right)$ can be stated as follows.

a) Magnitude of force of attraction or repulsion between any two charged particles never crosses $\left(c^{4} / G\right)$.

b) Magnitude of gravitational force of attraction between any two massive bodies never crosses $\left(c^{4} / G\right)$.

c) Magnitude of mechanical force on a revolving/rotating body never crosses $\left(c^{4} / G\right)$.

d) Magnitude of electromagnetic force on a revolving body never crosses $\left(c^{4} / G\right)$.

\subsection{Simple applications of $\left(c^{5 / G}\right)$ can be stated as fol-} lows.

a) Mechanical power never crosses $\left(c^{5} / G\right)$

b) Electromagnetic power never crosses $\left(c^{5} / G\right)$

c) Thermal radiation power never crosses $\left(c^{5} / G\right)$

d) Gravitational radiation power never crosses $\left(c^{5} / G\right)$

\section{To derive the Planck mass}

So far no theoretical model proposed a derivation for the Planck mass. Two derive the Planck mass the following two conditions can be given a chance.

Assuming that gravitational force of attraction between two Planck particles of mass $\left(\mathrm{M}_{\mathrm{Pl}}\right)$ separated by a minimum distance $\left(r_{\min }\right)$ be,

$\left[\frac{G M_{P l} M_{P l}}{r_{\min }^{2}}\right] \equiv\left(\frac{c^{4}}{G}\right)$

With reference to wave mechanics, let

$2 \pi \cdot r_{\text {min }} \cong \lambda_{P l}=\left[\frac{h}{c \cdot M_{P l}}\right]$

Here, $\lambda_{P l}$ represents the wavelength associated with the Planck mass. With these two assumed conditions Planck mass can be obtained as follows.

$M_{P l}=\sqrt{\frac{h c}{2 \pi G}} \cong \sqrt{\frac{\hbar c}{G}}$

\section{Schwarzschild radius of a black hole}

The 4 basic physical properties of a rotating black hole are its mass, size, angular velocity and temperature. Without going deep into the mathematics of black hole physics in the following subsections an attempt is made to understand and fit the black hole radius and temperature.

In all directions if a force of magnitude $\left(c^{4} / G\right)$ acts on the massenergy content of the assumed celestial body it approaches a minimum radius of $\left(G M / c^{2}\right)$ in the following way. Origin of the fore $\left(c^{4} / G\right)$ may be due to self-weight or internal attraction or external compression.

$R_{\min } \cong \frac{M c^{2}}{\left(c^{4} / G\right)} \cong \frac{G M}{c^{2}}$

If no force (of zero magnitude) acts on the mass content $M$ of the assumed massive body, its radius becomes infinity.

With reference to the average magnitude of $\left(0, \frac{c^{4}}{G}\right) \cong \frac{c^{4}}{2 G}$, the presently believed Schwarzschild radius can be obtained as

$(R)_{\text {ave }} \cong \frac{M c^{2}}{\left(c^{4} / 2 G\right)} \cong \frac{2 G M}{c^{2}}$

This proposal is very simple and seems to be different from the existing concepts of General theory of relativity.

\section{Temperature of a growing Planckion or evolving black hole}

At any time, the growing Planckion's thermal energy density can be estimated with the following assumption or conjecture. With this (classical) conjecture, Hawking's quantum mechanical black hole temperature formula (Hawking SW 1975) for Planck mass can be obtained. To prove it, it is a must to measure the mass, size and temperature of any black hole. With reference to the current comic microwave back ground temperature and current Hubble length it can be verified!

$a T_{t}^{4}\left(\frac{4 \pi}{3} R_{t}^{3}\right) \cong \frac{M_{t} c^{2}}{5760 \pi}$

Here, $\left(M_{t}, R_{t}, T_{t}\right)$ represent the instantaneous mass, radius and temperature of the growing black hole or growing Planckion respectively. With respect to the compound radiation constant $a \cong \frac{\pi^{2}}{15} \frac{k_{B}^{4}}{\hbar^{3} c^{3}}$ and the Planck mass $M_{P l} \cong \sqrt{\frac{\hbar c}{G}}$, above relation takes the following simple form.

$T_{t}=\frac{\hbar c^{3}}{8 \pi k_{B} G \sqrt{M_{P l} M_{t}}}$

Here, $M_{P l}$ represents the baby Planckion mass. It is similar to the expression derived by S.W. Hawking for the black hole temperature with a change in the mass of the black hole. According Hawking, temperature of a black hole is given by the following famous relation.

$T_{B}=\frac{\hbar c^{3}}{8 \pi k_{B} G M_{B}}$

Here, $\left(M_{B}, T_{B}\right)$ represent the mass and temperature of the black hole respectively. Note that, so far Hawking's proposal is not verified and not confirmed by any of the advanced astrophysical observations or Large Hadron Collider experiments (Steven B. Giddings et al 2002, F. Belgiorno et al 2010). It is being believed based on the advanced quantum mechanical theoretical and mathematical formulations. Now, above relation can be re-expressed as follows.

$T_{t}=\sqrt{\frac{M_{t}}{M_{P}}} \cdot\left(\frac{\hbar c^{3}}{8 \pi k_{B} G M_{t}}\right)$

At the Planck scale,

$T_{P l}=\frac{\hbar c^{3}}{8 \pi k_{B} G M_{P l}}$ 
Here, $T_{P l}$ represents the baby Planckion temperature. At Planck scale, proposed view of black hole temperature and Hawking's view of black hole temperature seems to be the same. Baby Planckion mass possesses a very high temperature and can be considered as a very hot fire ball.

\section{Understanding the origin of galactic black hole masses}

Now it can be suggested that, beginning from the Planck scale, high temperature Planckion is growing by absorbing the vacuum energy. During its evolution as it is absorbing the vacuum energy, its mass and size both will increase and hence its temperature will decrease. As its temperature decreases, its vacuum energy absorption capacity decreases and hence its growth rate decreases with increasing mass or decreasing temperature. During cosmic evolution, as time passes, Planckion will grow and its growth rate decreases with its decreasing temperature. Very high temperature of the baby Planckion is the heat source for the evolving Planckion temperature. Note that, there is no other internal mechanism for the assumed temperature of any evolving black hole or evolving Planckion. Proceeding further, growing Planckion can be considered as the seed and center of any galaxy. With reference to the Compton wavelength of nucleon and electron two semi stable massive Planckions can be estimated in the following.

\section{Case 1: Nucleon and its Compton wave length}

During cosmic evolution, as time passes at one particular instant, mass density of the growing Planckion approaches the nucleon and its Compton wavelength. Clearly speaking,

$\left[M_{t} c^{2}\left(\frac{4 \pi}{3} R_{t}^{3}\right)^{-1}\right] \Rightarrow m_{n} c^{2}\left(\frac{4 \pi}{3}\left(\frac{\hbar}{m_{n} c}\right)^{3}\right)^{-1}$

Where $m_{n}$ represents the mass of nucleon. If so the mass of the heavy Planckion can be obtained as follows.

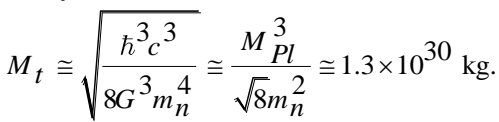

This can be compared with the current estimates of black hole masses and the Chandrasekhar mass limit $\cong 2.88 \times 10^{30} \mathrm{~kg}$ ( $\mathrm{S}$. Chandrasekhar 1931). From relation (7) its corresponding temperature can be estimated to be $7.32 \times 10^{11} \mathrm{~K}$. Note that this temperature is sufficient to heat the black hole's surrounding matter that is expected to emit $\mathrm{X}$-rays.

\section{Case 2: Electron and its Compton wave length}

During cosmic evolution, as time passes at one particular instant, mass density of the growing Planckion approaches the electron and its Compton wavelength. Clearly speaking,

$\left[M_{t} c^{2}\left(\frac{4 \pi}{3} R_{t}^{3}\right)^{-1}\right] \Rightarrow m_{e} c^{2}\left(\frac{4 \pi}{3}\left(\frac{\hbar}{m_{e} c}\right)^{3}\right)^{-1}$

Where $m_{e}$ represents the mass of electron. If so the mass of the heavy Planckion can be obtained as follows.

$$
M_{t} \cong \sqrt{\frac{\hbar^{3} c^{3}}{8 G^{3} m_{e}^{4}}} \cong \frac{M_{P l}^{3}}{\sqrt{8} m_{e}^{2}} \cong 4.38 \times 10^{36} \mathrm{~kg} .
$$

This can be compared with the current assumed galactic black hole masses. From relation (7) its corresponding temperature can be estimated to be $4.0 \times 10^{8} \mathrm{~K}$.

\section{Black hole cosmology - the true quantum gravity replica}

Combining quantum mechanics and general theory of relativity is a very typical issue and needs conceptual fine tuning and more sophisticated technology. By considering the subject of 'black hole cosmology' as a key branch of the quantum gravity, many fundamental issues of theoretical and observational cosmology can be understood. To consider the subject of black hole cosmology as the quantum gravitational cosmology it is imperative to consider the following two concepts.

1) To consider the current cosmic microwave back ground temperature as a quantum gravitational effect of the evolving primordial cosmic black hole automatically general theory of relativity and quantum mechanics can be combined into a 'scale independent' true unified model of quantum gravity. By considering the 'Planck mass' as the initial mass of the baby Hubble volume, past and current physical and thermal parameters of the cosmic black hole can be understood. Current rate of cosmic black hole expansion is being stopped by the microscopic quantum mechanical lengths. To understand the ground reality of current cosmic rate of expansion, sensitivity and accuracy of current methods of estimating the magnitudes of current CMBR temperature and current Hubble constant must be improved and alternative methods must be developed.

2) If it is true that galaxy constitutes so many stars, each star constitutes so many hydrogen atoms and light is coming from the excited electron of galactic hydrogen atom, then considering redshift as an index of 'whole galaxy' receding may not be reasonable. During cosmic evolution, at any time in the past, in hydrogen atom emitted photon energy was always inversely proportional to the CMBR temperature. Thus past light emitted from older galaxy's excited hydrogen atom will show redshift with reference to the current laboratory data. As cosmic time passes, in future, the absolute rate of cosmic expansion can be understood by observing the rate of increase in the magnitude of photon energy emitted from laboratory hydrogen atom. Aged super novae dimming may be due to the effect of high cosmic back ground temperature.

Considering the Planck scale, past, current and future thermal and physical parameters of the evolving universe can be quantified. Similarly by considering the Compton wavelength of proton or pion, current Hubble length can be fitted. To implement the Planck scale and Quantum mechanics in cosmology it is necessary to implement 'quantum gravity' in cosmology. One of the main difficulties of quantum gravity is that quantum gravitational effects are only expected to become apparent only near the Planck scale but not at ordinary energy scales. Present notion is that, "with current science and technology it is impossible to test the predictions of quantum gravity at low energy scales". Physics has proceeded by a series of "successive approximations" allowing more and more accurate predictions over a wider and wider range of phenomena. Anyhow if theoretical predictions are not in line with the observations, then either observations has to be interpreted in a different manner or theory has to be modified as per the observations. If one is willing to consider the evolving distance cosmic back ground as the base of quantum gravity, then automatically 'the scale' problem can be eliminated. Combining general relativity and quantum mechanics requires dynamic space-time structure with quantum effects at all energy scales. Procedure involved in this new approach can be understood in the following way.

1) If one is willing to consider the cosmic microwave back ground temperature as a quantum gravitational effect of the evolving universe one may be able to stand on the unified highway.

2) Moving further if one is willing to consider the CMBR temperature as the characteristic temperature of the evolving primordial cosmic black hole one may be able to run on the unified highway. 
3) Proceeding further if one is willing to consider the primordial cosmic black hole as an evolving and light speed rotating black hole with angular velocity identical with the Hubble constant, one may be able to identify the destiny on the unified highway.

4) Proceeding further and further, if one is willing to consider the current black hole universe as decelerating (as suggested by hot big bang model), one may be able to reach the destiny on the unified highway.

Thus based on the quantum gravitational back ground, it is possible to show that,

a) From the beginning of Planck scale, universe is a growing and light speed rotating black hole.

b) Considering the geometric mean mass of Planck mass and the current cosmic black hole mass - current cosmic back ground temperature can be estimated accurately.

c) Current rate of cosmic black hole expansion is being stopped by the microscopic quantum mechanical lengths.

d) As cosmic time passes, decreasing back ground cosmic black hole temperature makes hydrogen atom to emit increased quanta of energy causing the observed redshift. In future, with reference to current laboratory hydrogen atom, decreasing current cosmic temperature and measured rate of increase in emitted photon energy - true rate of (current and future) cosmic expansion can be understood.

e) Cosmic light speed rotation certainly leads to galactic revolution about the cosmic black hole center. Along with the mass of galaxy, galactic cosmological revolution speed plays a vital role in understanding the galaxy rotation curve. With reference to the MOND results (Milgrom, M 1983, Martin Bojowald 2012), and considering the galactic revolving speed $V_{g}$ about the center of the cosmic black hole (that rotates at light speed), rotational speed of a star in any galaxy can be represented as $v_{s} \cong \sqrt[4]{G M\left(V_{g} H_{0}\right)}$. Advantage of this proposal is that, qualitatively reasons for the constancy of the galactic rotational curves can be understood and by knowing the galactic mass and star's rotational speed, quantitatively galactic revolving speed and hence radial distance between galaxy and the cosmic black hole center can be estimated.

Proposed current cosmic deceleration can be understood and verified in the following way. Modern cosmologists believe that the rate of the change of the Hubble constant describes how fast/slow the Hubble constant changes over time and this rate does not tell if the Universe is currently expanding. This logic seems to be misleading. In authors opinion, if magnitude of past Hubble's constant was higher than the current magnitude then magnitude of past $\left(c / H_{t}\right)$ will be smaller than the current Hubble length $\left(c / H_{0}\right)$. If so rate of the decrease of the Hubble constant can be considered as a true index of rate of increase in Hubble length and thus with reference to Hubble length, rate of the decrease of the Hubble constant can be considered as a true index of cosmic rate of expansion. Proceeding further - in future, certainly with reference to current Hubble's constant, $d\left(c / H_{0}\right) / d t$ gives the true cosmic rate of expansion. Same logic can be applied to cosmic back ground temperature also. Clearly speaking $d\left(T_{0}\right) / d t$ gives the true cosmic rate of expansion. To understand the ground reality, sensitivity and accuracy of current methods of estimating the magnitudes of $\left(H_{0}\right.$ and $\left.T_{0}\right)$ must be improved. Need of new mathematical methods \& techniques, computer simulations, advanced engineering skills seem to be essential in this direction.

\section{Results and discussion}

\subsection{Important results}

a) Physical measurements of the black hole universe.
If it is assumed that, from the beginning of the Planck scale, universe always rotates at light speed with angular velocity identical to the corresponding Hubble constant,

At the Planck scale,

$R_{P l} \cong \frac{2 G M_{P l}}{c^{2}}$ and $H_{P l} \cong \frac{c}{R_{P l}} \cong \frac{c^{3}}{2 G M_{P l}}$

Where, $\left(M_{P l}, R_{P l}, H_{P l}\right)$ represent the Planck scale mass, radius and Hubble constant respectively. At any time in the past,

$R_{t} \cong \frac{2 G M_{t}}{c^{2}}$ and $H_{t} \cong \frac{c}{R_{t}} \cong \frac{c^{3}}{2 G M_{t}}$

Where, $\left(M_{t}, R_{t}, H_{t}\right)$ represent the past mass, radius and Hubble constant respectively. At present,

$R_{0} \cong \frac{2 G M_{0}}{c^{2}}$ and $H_{0} \cong \frac{c}{R_{0}} \cong \frac{c^{3}}{2 G M_{0}}$

Where, $\left(M_{0}, R_{0}, H_{0}\right)$ represent the past mass, radius and Hubble constant respectively.

b) Temperature of the evolving black hole universe At the Planck scale,

$T_{P l} \cong \frac{\hbar c^{3}}{8 \pi k_{B} G M_{P l}}$

Where, $T_{P l}$ represents the Planck scale cosmic black hole's temperature. At any time in the past,

$T_{t} \cong \frac{\hbar c^{3}}{8 \pi k_{B} G \sqrt{M_{t} M_{P l}}} \cong \frac{\hbar}{4 \pi k_{B}}\left(\frac{c^{3}}{2 G \sqrt{M_{t} M_{P l}}}\right)$

$\cong \frac{\hbar \sqrt{H_{t} H_{P l}}}{4 \pi k_{B}}$

Where, $T_{t}$ represents the past cosmic black hole's temperature. At present,

$T_{0} \cong \frac{\hbar c^{3}}{8 \pi k_{B} G \sqrt{M_{0} M_{P l}}} \cong \frac{\hbar}{4 \pi k_{B}}\left(\frac{c^{3}}{2 G \sqrt{M_{0} M_{P l}}}\right)$

$\cong \frac{\hbar \sqrt{H_{0} H_{P l}}}{4 \pi k_{B}}$

Where, $T_{0}$ represents the current cosmic black hole's temperature From this relation current Hubble's constant can be expressed and fitted in the following way.

$H_{0} \cong\left(\frac{4 \pi k_{B} T_{0}}{\hbar}\right)^{2} \frac{1}{H_{P l}} \cong\left(\frac{4 \pi k_{B} T_{0}}{\hbar}\right)^{2}\left(\frac{2 G M_{P l}}{c^{3}}\right)$

$\cong 66.893 \mathrm{~km} / \mathrm{sec} / \mathrm{Mpc}$.

This is matching with the current estimations of the Hubble's constant (David N. Spergel et al 2013): $\{(67.80 \pm 0.77),(68.1 \pm 1.2)\} \mathrm{km} / \mathrm{sec} / \mathrm{Mpc}$. Thus from now onwards, CMBR temperature can be called as 'Comic Black Hole's Thermal Radiation' temperature. If current rate of decrease in $H_{0}$ is small very and is beyond the scope of observational or experimental detection - for the whole cosmic black hole as $H_{0}$ practically remains constant, its corresponding thermal energy density will be the same throughout its volume. This may be the reason for the observed 'isotropic' nature of the current CMB radiation. At any time in the past,

$\frac{T_{t}}{T_{0}} \cong \sqrt{\frac{H_{t}}{H_{0}}}$

One interesting thing is that, at any given time,

$\frac{8 \pi G a T_{t}^{4}}{3 H_{t}^{2} c^{2}} \cong \frac{8 \pi G a}{3 c^{2}}\left(\frac{T_{t}^{4}}{H_{t}^{2}}\right) \cong \frac{8 \pi G a}{3 c^{2}}\left(\frac{h}{4 \pi k_{B}}\right)^{4}\left(\frac{H_{P l}^{2}}{16 \pi^{4}}\right)$ 
Based on this observation and with reference to the observed CMBR anisotropy, it is possible to guess that,

$$
\left.\begin{array}{l}
\frac{\delta T}{T_{t}} \cong\left(\frac{3 H_{t}^{2} c^{2}}{8 \pi G a T_{t}^{4}}\right)^{-1} T_{t} \cong 5.5262 \times 10^{-5} T_{t} \\
\frac{\delta T}{T_{0}} \cong\left(\frac{3 H_{0}^{2} c^{2}}{8 \pi G a T_{0}^{4}}\right)^{-1} T_{0} \cong 5.5262 \times 10^{-5} T_{0}
\end{array}\right\}
$$

At present, $\delta T \cong 133 \mu \mathrm{K}$. Qualitatively this can be compared with the current observed (David N. Spergel et al 2013) temperature fluctuations of $\delta T \cong 570 \mu \mathrm{K}$. From this it can be suggested that, at any time, the $\mathrm{CMB}$ anisotropy is directly proportional to the $\mathrm{CMB}$ temperature at that time. Alternatively it can be suggested that, at any time, $\mathrm{CMB}$ isotropy is inversely proportional to the $\mathrm{CMB}$ temperature at that time.

c) Matter density in the evolving black hole universe At the Planck scale,

$\rho_{P l} \cong\left(\frac{a T_{P l}^{4}}{c^{2}}\right)$

Where, $\rho_{P l}$ represents the Planck scale cosmic black hole's matter density. At any time in the past,

$\rho_{t} \cong\left[1+\ln \left(\frac{M_{t}}{M_{P l}}\right)\right]\left(\frac{a T_{t}^{4}}{c^{2}}\right) \cong\left[1+\ln \left(\frac{H_{P l}}{H_{t}}\right)\right]\left(\frac{a T_{t}^{4}}{c^{2}}\right)$

Where, $\rho_{t}$ represents the past cosmic black hole's matter density. At present,

$$
\begin{gathered}
\rho_{0} \cong\left[1+\ln \left(\frac{M_{0}}{M_{P l}}\right)\right]\left(\frac{a T_{0}^{4}}{c^{2}}\right) \cong\left[1+\ln \left(\frac{H_{P l}}{H_{0}}\right)\right]\left(\frac{a T_{0}^{4}}{c^{2}}\right) \\
\cong 6.53 \times 10^{-32} \mathrm{gram} / \mathrm{cm}^{3}
\end{gathered}
$$

Where, $\rho_{0}$ represents the current cosmic black hole's matter density. Note that almost (70 to 80 ) \% of the galaxies is in the form of elliptical and spiral galaxies. For spiral galaxies, mass-to-light ratio is $\eta \mathrm{h}_{0}^{-1} \cong 9 \pm 1$ and for elliptical galaxies $\eta \mathrm{h}_{0}^{-1} \cong 10 \pm 2$. For our galaxy inner part $\eta \mathrm{h}_{0}^{-1} \cong 6 \pm 2$. Thus the average $\eta \mathrm{h}_{0}^{-1}$ is very close to 9 . Based on the average mass-to-light ratio for elliptical and spiral galaxies present matter density can be expressed with the following relation.

$\left(\rho_{m}\right)_{0} \cong 1.5 \times 10^{-32} \eta h_{0} \mathrm{gram} / \mathrm{cm}^{3}$

Here, $\eta \cong\left\langle\frac{M}{L}\right\rangle_{\text {galaxy }} /\left\langle\frac{M}{L}\right\rangle_{\text {sun }}$ and $h_{0} \cong 0.68$.

Corresponding matter density is close to $6.24 \times 10^{-32} \mathrm{gram} / \mathrm{cm}^{3}$ and can be compared with the above proposed magnitude of $6.5 \times$ $10^{-32} \mathrm{gram} / \mathrm{cm}^{3}$.

d) Galactic rotational curves in the current black hole universe.

With reference to the MOND results, empirically rotational speed of a star is being represented as

$v_{s} \cong \sqrt[4]{G M a_{0}}$

Where $a_{0} \cong(1.2 \pm 0.3) \times 10^{-10} \mathrm{~m} \cdot \mathrm{sec}^{-2} \approx c H_{0} / 2 \pi$, and $M$ is the mass of galaxy. In the light speed rotating black hole universe,

1) The acceleration constant $a_{0}$ is not a constant but a variable and depends on the galactic revolving speed about the center of the light speed rotating black hole universe.

2) Its magnitude can be assumed to be proportional to the current Hubble constant and can be called as the cosmological galactic acceleration.
3) By considering the galactic revolving speed $V_{g}$ about the center of the cosmic black hole, magnitude of $\left(\mathrm{cH}_{0}\right)$ can be assumed to vary as

$\left(V_{g} / c\right)\left(c H_{0}\right) \cong\left(V_{g} H_{0}\right) \cong a_{g} \cong r_{g} H_{0}^{2}$.

where $r_{g}$ is the distance between the galaxy and the cosmic center. Thus authors replace the empirical acceleration constant $a_{0}$ with (a variable) cosmological galactic acceleration, $a_{g} \cong V_{g} H_{0}$. Now rotational speed of a star in any galaxy can be represented as follows.

$$
v \cong \sqrt[4]{G M\left(\mathrm{~V}_{g} H_{0}\right)} \cong \sqrt[4]{G M r_{\mathrm{g}} H_{0}^{2}}
$$

Here if it is assumed that, galaxies under observation possesses a cosmological revolving speed in the range 0.1 to 0.25 times the speed of light currently observed all galactic rotational speeds can be fitted well. If current $H_{0} \cong 68 \mathrm{~km} / \mathrm{sec} / \mathrm{Mpc}$, $c H_{0} \cong 6.8 \times 10^{-10} \mathrm{~m} \cdot \mathrm{sec}^{-2}$ and $0.1\left(c H_{0}\right) \cong 0.66 \times 10^{-10} \mathrm{~m} \cdot \mathrm{sec}^{-2}$ $0.25\left(\mathrm{cH}_{0}\right) \cong 1.65 \times 10^{-10} \mathrm{~m} \cdot \mathrm{sec}^{-2}$. Advantage of this proposal is that, by knowing the galactic mass and rotational speeds of its stars, galactic revolving speed and hence distance between galaxy and the cosmic black hole center can be estimated. This is for further study. It is true that this proposal is

1) Qualitatively suitable for understanding the galactic rotation curves in the light of light speed cosmic rotation.

2) By knowing the galactic rotational speeds quantitatively suitable for estimating the galactic cosmological revolution speed and distance from the cosmic center.

e) Galactic redshift in the evolving black hole universe.

Observed galactic redshift can be understood in the following way. $\left.\begin{array}{l}\frac{E_{0}}{E_{t}} \cong \frac{\lambda_{t}}{\lambda_{0}} \cong \frac{T_{t}}{T_{0}} \cong\left(z_{0}+1\right) \\ z_{0} \cong \frac{E_{0}-E_{t}}{E_{t}} \cong \frac{\lambda_{t}-\lambda_{0}}{\lambda_{0}} \cong \frac{T_{t}-T_{0}}{T_{0}}\end{array}\right\}$

Here, $E_{t}$ is the energy of emitted photon from the galactic hydrogen atom and $E_{0}$ is the corresponding energy in the laboratory. $\lambda_{t}$ Is the wave length of emitted and received photon from the galactic hydrogen atom and $\lambda_{0}$ is the corresponding wave length in the laboratory. $T_{t}$ is the cosmic temperature at the time when the photon was emitted and $T_{0}$ is the current cosmic temperature and $z_{0}$ is the current redshift.

At any time in the past - in support of the proposed cosmological red shift interpretation, in hydrogen atom, total energy of electron in $\mathrm{n}^{\text {th }}$ orbit can be expressed as follows.

$$
\begin{aligned}
\left(E_{\text {tot }}\right)_{t} & \cong-\left(\frac{T_{0}}{T_{t}}\right) \frac{e^{4} m_{e}}{32 \pi^{2} \varepsilon_{0}^{2} \mathrm{~h}^{2} n^{2}} \\
& \cong-\sqrt{\frac{H_{0}}{H_{t}}} \cdot \frac{e^{4} m_{e}}{32 \pi^{2} \varepsilon_{0}^{2} \mathrm{~h}^{2} n^{2}}
\end{aligned}
$$

Where $n=1,2,3, .$. From laboratory point of view, above concept can be understood in the following way. After some time in future, $z_{f} \cong \frac{E_{f}-E_{0}}{E_{0}} \cong \frac{E_{f}}{E_{0}}-1$

Here, $E_{f}$ is the energy of photon emitted from laboratory hydrogen atom after some time in future. $E_{0}$ Is the energy of current photon emitted from laboratory hydrogen atom. $z_{f}$ is the redshift of laboratory hydrogen atom after some time in future. In future within the scope of experimental accuracy of laboratory hydrogen atoms redshift - $\left[d\left(z_{f}\right) / d t\right]$ can be considered as a true index of absolute rate of cosmic expansion. It can be understood from table-1 in the following way. 
Table 1: To Understand the True Nature of Cosmic Expansion

\begin{tabular}{cll}
\hline & Nature of change & Nature of cosmic expansion \\
\hline \multirow{2}{*}{$\frac{d\left(z_{f}\right)}{d t}$} & Increasing & Acceleration \\
& Constant & Uniform rate \\
& Decreasing & Deceleration \\
& Zero & Zero \\
\hline
\end{tabular}

f) To understand the galactic revolution and receding.

As the universe is growing and always rotating at alight speed, at any time, any galaxy will have revolution speed as well as receding speed simultaneously and both can be expressed in the following way.

$\left(V_{g}\right)_{\text {revolution }} \cong\left(\frac{r_{g}}{R_{t}}\right) c \cong r_{g} H_{t}$

$r_{g}$ Is the distance between galaxy and the cosmic center, $R_{t}$ is the cosmic radius at time $t$ and $r_{g} \leq\left(R_{t} \cong \frac{c}{H_{t}}\right)$.

$$
\left(v_{g}\right)_{\text {receding }} \cong\left(\frac{r_{g}}{R_{t}}\right) \frac{d R_{t}}{d t} \cong\left(\frac{r_{g}}{R_{t}}\right) \frac{d}{d t}\left(\frac{c}{H_{t}}\right)
$$

At present,

$$
\begin{aligned}
& \left(V_{g}\right)_{\text {revolution }} \cong\left(\frac{r_{g}}{R_{0}}\right) c \cong r_{g} H_{0} \\
& \left(v_{g}\right)_{\text {receding }} \cong\left(\frac{r_{g}}{R_{0}}\right) \frac{d R_{0}}{d t} \cong\left(\frac{r_{g} H_{0}}{c}\right) \frac{d}{d t}\left(\frac{c}{H_{0}}\right)
\end{aligned}
$$

g) Strange microscopic quantum mechanical result.

To a great surprise it is noticed that,

$$
\sqrt{\left(\frac{2 G M_{0}}{c^{2}}\right)\left(\frac{2 G \sqrt{m_{p} m_{e}}}{c^{2}}\right)} \cong \frac{e^{2}}{4 \pi \varepsilon_{0} m_{e} c^{2}}
$$

Here, $m_{e}, m_{p}$ represent the rest masses of electron and proton respectively and $M_{0} \cong c^{3} / 2 G H_{0}$. if one is willing to consider that the current black hole universe is decelerating and reaching a point of no expansion, i.e. saturation of expansion, above relation can be expressed as follows.

$$
\frac{2 G \sqrt{M_{S} \sqrt{m_{p}^{m_{e}}}}}{c^{2}} \Rightarrow \frac{e^{2}}{4 \pi \varepsilon_{0} m_{e} c^{2}}
$$

Where $M_{S} \cong c^{3 / 2 G H_{S}}$ and $H_{S}$ can be called as the saturated Hubble constant. If so to a great accuracy, $H_{S} \cong 67.53 \mathrm{~km} / \mathrm{sec} / \mathrm{Mpc}$. Proceeding further, if one is willing to consider 'half the magnitude of classical radius of electron' as the Compton wavelength of charged Pion (H. Yukawa 1935), then

$$
\frac{G \sqrt{M_{S} \sqrt{m_{p}^{m_{e}}}}}{c^{2}} \Rightarrow \frac{\hbar}{m_{\pi^{c}}}
$$

If so, $H_{S} \cong 67.0 \mathrm{~km} / \mathrm{sec} / \mathrm{Mpc}$. clearly speaking, when the magnitude of Hubble constant reaches $67 \mathrm{~km} / \mathrm{sec} / \mathrm{Mpc}$, quantum mechanically rate of expansion of the black hole universe becomes zero and there may be no further expansion! This is an interesting result of the combined study of black hole cosmology and quantum mechanics.

\subsection{Discussion}

Please note that, so far no ground based experiment directly confirmed.

a) The Hubble's redshift based increase in photon wavelength/loss in photon energy.

b) The actual galaxy receding speed and galaxy acceleration as per the Hubble's law. c) The existence of dark energy or dark matter. (Clearly speaking nobody is sure about the detection of dark energy and dark matter with the known experimental techniques).

d) The basic physically observable characteristics of dark energy. Similarly no ground based experiment directly confirmed the basic physically observable characteristics of dark matter.

e) The current magnitudes and applications of dark matter content, dark energy content and the observable cosmic matter content. Thus so far no body quantified the distance cosmic back ground.

Not only that, so far

f) Nobody explained the real picture of big bang, nobody quantified the big bang and nobody considered the point of big bang as a characteristic reference point of cosmic expansion in all directions.

g) Nobody explained the role of dark energy/dark matter in the primordial nucleo-synthesis.

h) No theoretical/experimental proof is available for cosmic nonrotation. Similarly no theoretical proof is available for cosmic or celestial bodies super luminal rotational speeds.

i) No cosmic parameter has been obtained from the microscopic physics theoretically.

j) Hawking's black hole temperature formula (in its derived form) is not verified and not confirmed by any of the advanced astrophysical observations or Large Hadron Collider experiments.

k) No theoretical model or no experimental result disproved the model of black hole cosmology.

\section{Conclusion}

Based on the above concepts, logics, results and data fitting- it can be suggested that,

1) Along with presently the believed black holes that are expected to be formed by gravitational collapse of a massive star, there may exist primordial evolving black holes.

2) During their evolution, by absorbing the hidden vacuum energy primordial evolving black holes become massive.

3) Planck mass can be considered as the basic mass of any black hole and can be called as the baby Planckion. Very high temperature of the baby Planckion is the only heat source for the evolving black holes or evolving Planckion's decreasing temperature.

4) Evolving black hole or evolving Planckion temperature is inversely proportional to the square root of the baby Planckion mass and its evolving mass.

5) Any evolving black hole or evolving Planckion is a hot fire ball and can emit electromagnetic radiation.

6) Current cosmic microwave back ground temperature can be considered as the quantum gravitational effect of the evolving primordial cosmic black hole and thus starting from the Planck scale, the foundations of Quantum mechanics and General theory of relativity may be reviewed in a unified manner.

7) Observed galactic redshift and distant super novae dimming, both can be considered as the results of past high cosmic temperature dependent light emission mechanism and by considering the future redshift of the 'laboratory' hydrogen atom, the true rate of future cosmic expansion can be understood. To understand the ground reality of the future cosmic rate of expansion, sensitivity and accuracy of current methods of estimating the magnitudes of $\left(H_{0}\right.$ and $\left.T_{0}\right)$ must be improved.

8) Considering the light speed rotation of the cosmic black hole, galactic rotational curves can be understood and there by the concept of 'dark matter' can be relinquished.

9) Until the confirmation of right cosmology, black hole cosmology (Pathria, R. K 1972, Good, I. J 1972, Smolin, L 2004, Zhang, Tianxi 2010, Andy Gardner et al 2010, Chul-Moon Yoo et al 2013, Michael E. McCulloch 2014, Poplawski, N. J 2014, U. V. S. Seshavatharam 2014) can be given equal priori- 
ty along with the presently believed standard cosmology and there by the concept of 'dark energy' can be relinquished.

10) A recurrent criticism of inflation is that the invoked inflation field does not correspond to any known physical field and that its potential energy curve seems to be an ad hoc means to accommodate almost any data obtainable. Anyhow, with reference to the past and current Hubble volumes, and by comparing the concepts, results and outcomes that can be obtained with the proposed model of black hole cosmology, in coming future, the need of inflation can be reviewed.

\section{Acknowledgements}

The first author is indebted to professor K. V. Krishna Murthy, Chairman, Institute of Scientific Research on Vedas (I-SERVE), Hyderabad, India and Shri K. V. R. S. Murthy, former scientist IICT (CSIR) Govt. of India, Director, Research and Development, I-SERVE, for their valuable guidance and great support in developing this subject.

\section{References}

[1] Hubble, E.P, PASP, 59, pp153-167, (1947). http://dx.doi.org/10.1086/125931.

[2] Hawking S.W. A Brief History of Time. Bantam Dell Publishing Group. (1988)

[3] Alpher R.A, bethe H, G. Gamow. Phys. rev.73, 80 (1948).

[4] A. Penzias. Nobel lecture. (1978).

[5] Steinhardt, Paul J. Scientific American.;304(4) pp. 18-25 (2011).

[6] Saul Perlmutter. Nobel lecture. (2011).

[7] David N. Spergel, Raphael Flauger and Renne Hlozek. arxiv: $1312.3313 \mathrm{v} 1$.

[8] Ashtekar, Abhay Physical Review Letters 57 (18): 2244-2247 (1986).

[9] Carlo Rovelli. Class.Quant.Grav.28:114005 (2011) http://dx.doi.org/10.1088/0264-9381/28/11/114005.

[10]Hawking, Stephen W. Quantum cosmology. In Hawking, Stephen W.; Israel, Werner. 300 Years of Gravita-tion. Cambridge University Press. pp. 631-651. (1987).

[11]Schwarz, John H. Progress of Theoretical Physics Supplement 170: 214-226 (2007) http://dx.doi.org/10.1143/PTPS.170.214.

[12]Maité Dupuis, James P. Ryan and Simone Speziale. A Short Review. SIGMA 8, 052, 31 pages. (2012).

[13]Hawking S.W. Commun. Math. Phys., v.43, 199-220. (1975) http://dx.doi.org/10.1007/BF02345020.

[14]Hawking SW. arXiv: 1401.5761v1 (2014).

[15]Mitra A. Foundations of Physics Letters.13:543-579. (2000) http://dx.doi.org/10.1023/A:1007810414531.

[16] Steven B. Giddings and Scott Thomas. Phys.Rev. D65 056010 (2002) http://dx.doi.org/10.1103/PhysRevD.65.056010.

[17]Belgiorno, S. L. Cacciatori, M. Clerici, V. Gorini, G. Ortenzi, L. Rizzi, E. Rubino, V. G. Sala, and D. Faccio. Phys. Rev. Lett. 105, 203901 (2010) http://dx.doi.org/10.1103/PhysRevLett.105.203901.

[18]S. Chandrasekhar, Philosophical Magazine (7th se-ries) 11 p.592-596 (1931).

[19]Milgrom, M. Astrophys. J. 270, 365-370. (1983). http://dx.doi.org/10.1086/161130.

[20]Martin Bojowald, Class. Quantum Grav. 29, 213001 (2012) http://dx.doi.org/10.1088/0264-9381/29/21/213001.

[21]H. Yukawa. Proc. Phys. Math. Soc. Jap. 17 (48). 1935.

[22]Pathria, R. K. Nature 240 (5379):298299 (1972). http://dx.doi.org/10.1038/240298a0.

[23]Good, I. J. Physics Today 25 (7): 15. (1972). http://dx.doi.org/10.1063/1.3070923.

[24]Smolin, L. Physica A 340, 705-713. (2004) http://dx.doi.org/10.1016/j.physa.2004.05.021.

[25]Zhang, Tianxi. Astrophysics and Space Science, Vol-ume 330, Issue 1, pp 157-165. (2010) http://dx.doi.org/10.1007/s10509-010-0372-4.

[26] Andy Gardner, Joseph P. Conlon. Complexity. Vol.18, Issue 5, pp4856. (2013) http://dx.doi.org/10.1002/cplx.21446.

[27]Chul-Moon Yoo, Hirotada Okawa, Ken-ichi Nakao. Phys. Rev. Lett. $111,161102(2013)$ http://dx.doi.org/10.1103/PhysRevLett.111.161102

[28]Michael E. McCulloch. Galaxies, 2, 81-88, (2014). http://dx.doi.org/10.3390/galaxies2010081.

[29]Poplawski, N. J. Class. Quantum Grav. 31, 065005 (2014) http://dx.doi.org/10.1088/0264-9381/31/6/065005.
[30]U. V. S. Seshavatharam, S. Lakshminarayana. Physical Science International Journal, Vol-4, Issue-6, p.842-879. (2014). 